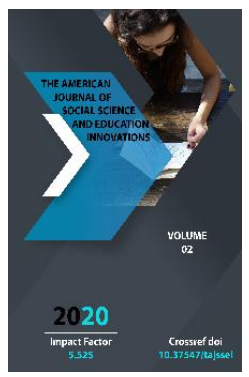

\title{
Delimitation Of Computer Information From Other Documents And Material Evidence
}

Journal Website: http://usajournalshub.c om/index,php/tajssei

\section{Ulzana Rakhimova \\ Lecturer at the Department of Criminal Procedure Law, Tashkent State Law University, Uzbekistan}

\section{ABSTRACT}

The article examines some aspects concerning the essence of computer information as evidence in a criminal case and ways of securing it as such, as well as issues regarding the differentiation of computer information from other documents and material evidence.

\section{KEYWORDS}

Computer information, evidence in criminal case, material evidence electronic document, other documents.

\section{INTRODUCTION}

The Internet covers our life every day. As of January 2020, Northern Europe ranked first with 95 percent online penetration by region of the world, followed by Western Europe with 92 percent. The global average penetration rate was 59 percent, up from 35 percent in 2013[1]. In this regard, the so-called cybercrimes committed with the help of information technology have become more frequent. Every year, criminals become more sophisticated and commit "smart" crimes that require certain skills and abilities rather than physical strength. This became especially true during the coronavirus pandemic, when one third of the world's population was forced to stay at home. However, the crime rate did not fall.

As the basis of modern society, computer information, in turn, acts as an inseparable element of social relations. In this regard, the question arises about the differentiation of computer information from other documents and material evidence.

The term "computer information" is disclosed in the Agreement on Cooperation of the CIS Member States in the Fight against Crimes in the Field of Computer Information, according to which "computer information" means 
"information in the memory of a computer, on machine or other media in a form that is accessible to the perception of a computer, or transmitted through communication channels "[2]. Thus, the main feature of computer information is the possibility of its perception by electronic computing (computer) equipment or transmission through communication channels.

The legislator does not secure computer information as a separate type of evidence in a criminal case. In this regard, two questions arise: is it possible to classify computer information as one of the types of evidence provided by the legislator? If not, is it possible to single out it as a separate type of proof?

When looking for an answer to the first question, it should be noted that most of all computer information meets the requirements of material evidence or other documents.

As evidence can be both official documents (certificates, acts, etc.) and unofficial (for example, a personal letter). Documents can be original (originals) and derivatives (copies). The documents must be obtained in accordance with the procedure established by law - seized in the course of any investigative action, requested or presented by any of the participants in the proceedings. The fact that they were received by the investigator or the court must be duly formalized. Official documents must contain all the necessary details (stamp, signatures, etc.).

A prerequisite for using documents as evidence is that their source must be known the author or performer. This is especially true for personal documents, which may not even contain a signature. Therefore, when checking such documents, an interrogation of their author or performer should be carried out, if necessary, handwriting or authorship examination can be carried out, and in relation to phonograms - phonoscopic. Therefore, the source of proof in relation to another document is the author of the document. The legal status of the specified source of evidence is determined by the competence of the author of the document, which is limited by the scope of his functions. Documents can be official when they come from government agencies, organizations. As other documents, acts of audits and documentary checks should be considered if the circumstances and facts, certified or stated in them, are important for the case. Revisions, documentary inspections can be appointed and carried out both in connection with the proceedings on a criminal case, and independently of it. Citizens can also be authors of documents. They usually issue documents that are various kinds of contracts, powers of attorney, receipts and other similar acts related to the exercise by citizens of their rights and obligations. A document emanating from an incompetent body, an official, from an inappropriate citizen is not evidence.

It is fundamentally important to find out who is the author of computer information (electronic document). There is no unambiguous interpretation of this issue in the scientific literature. To clarify the true nature of information and the essence of information processes that underlie the knowledge of a crime event, Polevoy N.A. proposes to proceed "from a close connection with reflection as a property of all matter, from the basic principles of Lenin's theory of reflection". Distinguishing the concepts of "reflection" and "display", he came to the conclusion that information is something that can be perceived by a cognizing subject or technical device, and thus it is "as if" separated "from its primary source - the mapping of the source of the object of knowledge" ; it can be "transferred in space, saved in time, transferred to another cognizing subject or technical device, for 
example a computer, subjected to other operations, the totality of which is called information processes"[3,p 61].

The most correct approach in distinguishing one species from another should take into account not one decisive feature, but a set of such features.

In order to understand the essence of evidence, it is necessary to find out the mechanism of their formation, including the patterns of trace formation and the procedural conditions for collection.

Kudryavtseva A.V. notes: "The introduction of a new source (type), means of proof must be conditioned by two criteria that must act simultaneously: 1) the mechanism for generating information in a particular source (it is the verification of the mechanism that gives an idea of the adequacy of the information reflected by the evidence); 2) a way of converting this information with the help of legal proceedings into evidence"[4]. Let's consider the mechanism of formation of a "other" document and computer information. "Other" documents include any documents not prepared in the course of procedural activities (certificates, acts of audits, audits and other checks, statements, receipts, etc.).

According to Article 85 of the Criminal Procedure Code of the Republic of Uzbekistan, proof consists in the collection, verification and assessment of evidence in order to establish the truth about the circumstances that are important for the legal, reasonable and fair resolution of the case.

Evidence is collected through the production of investigative and judicial actions: interrogation of the suspect, accused, defendant, witness, victim, expert; confrontation; presentation for identification; verification of testimony at the scene; notches; searches; inspections; surveys; exhumation of a corpse; experiments; obtaining samples for expert research; appointing examinations and audits; acceptance of submitted items and documents; listening to negotiations conducted from telephones and other intercom, as well as by conducting operational-search activities.

The most common are written documents (printed and handwritten). But the information contained in the document could be recorded in a different form. Such documents include materials of photography and filming, audio and video recordings and other media.

As evidence can be both official documents (certificates, acts, etc.) and unofficial (for example, a personal letter). Documents can be original (originals) and derivatives (copies). The documents must be obtained in accordance with the procedure established by law - seized in the course of any investigative action, requested or presented by any of the participants in the proceedings. The fact that they were received by the investigator or the court must be duly formalized.

Official documents must contain all the necessary details (stamp, signatures, etc.).

A prerequisite for the use of documents as evidence is that their source must be known the author or performer. This is especially true for personal documents, which may not even contain a signature. Therefore, when checking such documents, an interrogation of their author or performer should be carried out, if necessary, handwriting or authorship examination can be carried out, and in relation to phonograms - phonoscopic [5].

So, the author of the document acts as the source of proof in relation to the "other" document. The legal status of the specified source of evidence is determined by the competence of the author of the document, which is limited by the scope of his functions. Documents can be official when they come 
from government agencies, organizations. The acts of audits and documentary inspections should also be considered as "other" documents, if the circumstances and facts, certified or stated in them, are important for the case. Audits, documentary checks can be appointed and carried out both in connection with the criminal proceedings, and independently of it. Citizens can also be authors of documents. They usually issue documents that are various kinds of contracts, powers of attorney, receipts and other similar acts related to the exercise by citizens of their rights and obligations. A document emanating from an incompetent body, an official, from an inappropriate citizen is not evidence [6,p.214].

Thus, computer information is created using an algorithm given by the program. In turn, the program is created by a person. A computer does not know how to think, it only knows how to transform a set of signals selected in one way or another on the basis of a uniquely specified sequence of fixed operations. Since the process of data processing is carried out by technical means of computers (hardware and software), then we can talk about the emergence and receipt (perception) of information occurring indirectly through the "intellectual consciousness" of a person. Computer information (electronic document) is created and / or formed by a machine, but not by a person. When creating a text document, a computer program for generating an electronic document is implemented a priori on the machine. As we can see, the mechanism for the formation of a "different" document and computer information is different.

For criminal procedural use, computer information must be fixed on some medium. Semiletov S.I. emphasizes that an integral functional characteristic of any document is its ability to store and transmit information in time and space about events, facts, rights and obligations. And the main functional purpose of the document is associated with the process of fixing information, i.e. by the technological process of its fixation on a material carrier for the purpose of its longterm storage for subsequent use and use in public relations by presenting, viewing or transmitting a document in time and space. At the same time, he draws attention to the fact that both material carriers and methods of fixation can be very diverse; it is only important that the documents and the information recorded on them can be stored in time indefinitely, and the recording itself is carried out in compliance with the order corresponding to the functional purpose of each individual document. Such requirements provide a certain degree of protection of the document from possible forgeries and falsifications detected during visual inspection and evaluation of all available details of the document [7].

The main difference between an electronic document is the absence of a rigid binding to the carrier. More precisely, the same document can exist on different media, for example, in the form of electromagnetic waves (at the time of its transmission by radio or wire), while it remains authentic [8]. This difference, as noted by S.I. Semiletov, is determined by the peculiarities of the organization of electronic documents and the technology of fixing their content information. Features of information digital technology make it possible to instantly and repeatedly copy and transmit an electronic document through communication channels; it can be used simultaneously by an unlimited number of users [9].

One and the same electronic document can simultaneously exist in the form of an objective static record of a digital binary 
machine code, as a rule, in the form of a file with external attributes (name, extension, date of creation, volume) of one format or another on various electronic media. Moreover, a tangible medium, in contrast to a paper document, can be used repeatedly to record different documents (and not once). The file system for organizing the space of random access memory and media of such a complex electronic device as an electronic computer or computer is an important component that organizes the work and interaction of the processor, random access memory, interface buses and external devices. Another significant difference between computer information lies in the inability to directly and unambiguously perceive this information by the human senses. This is due to the fact that computer information exists in electronic digital form. And although it is located on a tangible medium, the very presence of computer information as well as its location on this medium cannot be determined without appropriate software and hardware.

A person perceives an electronic document as a named file with certain known attributes, the record of which is located in a certain, as a rule, known to the user place (first of all virtual, not physical) in the structure of organized catalogs of software and hardware. It should be noted that Gadasin V.A. and Konyavsky V.A., considering the systemic foundations of the difference between a written (analog) and an electronic document and referring to the "cardinal difference in the physical environment of the existence of a document", note that a traditional document is an analog display of information fixed on a hard medium and designed for direct perception by a subject - a person. The subject has knowledge, is capable of perceiving the graphical presentation of information, its "understanding" and processing on the basis of thinking. The basis of perception is a powerful image recognition apparatus, organically inherent in a person, which allows him to identify the components of a document with standards formed in consciousness. An electronic document is a digital display of information, the carrier of which is computer technology and informatics (VTI). An electronic document is directly perceived only by an object of an electronic digital environment - a hardware or software tool. Only an object of the electronic environment can process an electronic document: perform an a priori specified deterministic transformation of an input electronic document into an output electronic document. An electronic document only exists in an electronic (digital) environment of existence, while a written document exists in an analogue environment [10].

The practical significance of this distinction lies in the fact that for computer information and material evidence, the law provides for a different procedure for attaching to the case, storing and determining the further "fate" after a verdict or decision to terminate a criminal case.

\section{RESULTS}

It must be said that different researchers consider both the problem of including digital information in general in the current system of evidence, and the problem of including electronic documents. At the same time, positions are traced according to which, if computer information can fit into a generally accepted system of evidence, then for other objects of digital information, for example, computer programs, such an assignment will be questionable.

A.V. Tkachev draws attention to the fact that such features of electronic documents do not affect either the essence of evidence or their 
procedural function, therefore electronic documents fit well within the traditional system of evidence, subject to legal regulation of their specific features [11, p.130].

So, the following grounds are highlighted for distinguishing between computer information and physical evidence:

- by evidentiary value. Physical evidence is an item and the evidentiary value is determined by the physical properties or location of that item. Computer information is the content of information. This information is located on a tangible medium, otherwise it cannot be recognized as evidence, but the appearance of this medium does not in any way reflect the information that is recorded on it. It is the information itself that has evidentiary value, not its carrier;

- by the mechanism of education. Physical evidence is characterized by a mechanical elementary reflection of facts. The mechanism for the formation of computer information is determined by an algorithm that is specified by the developer (development team) and is implemented in a specific program, which is a means of reflecting facts;

- on the basis of perception. In material evidence, information is contained in its natural, uncoded form, and its transformation for perception is not necessary. Computer information is always mediated through a machine information carrier, outside of which it cannot exist, and its perception (computer information) is possible only through a technical means (computer);

- based on the environment of existence. Physical evidence is part of the analogue environment. Computer information is an environment of software and hardware, that is, an electronic environment.
Thus, in our opinion, the value of computer information as a source of evidence lies in the fact that the fixation of information in a given source occurs without processing it by the human consciousness, but in the form in which it objectively existed, regardless of subjective perception (who fixes it ). The foregoing provides a basis for the conclusion about the great value of this information and the need for more effective use of it in the criminal process, for which it is necessary to single out computer information as an independent source of evidence.

\section{REFERENCES}

1. https://www.statista.com/statistics/26932 9/penetration-rate-of-the-internet-byregion/

2. Agreement on cooperation of the CIS member states in the fight against crimes in the field of computer information // Information Bulletin of the Council of Heads of State and the Council of Heads of Government of the CIS. No. 1 (37). http://cis.minsk.by/page/866

3. Polevoy N.S. Forensic cybernetics. - M., 1989.- p. 61.

4. Kudryavtseva A.V. The concept of the theory of proof in the light of the problem of the unity of the process // Actual problems of law in Russia and the CIS countries - 2006: materials of the VIII International scientific and practical conference. -Chelyabinsk, 2006.

5. Lupinskaya P.A. Criminal Procedure Law of the Russian Federation. - M., 2005.

6. Bozhiev V.P. Criminal process. - M., 2002 .p. 214.

7. Semiletov S.I. Electronic document as a product of the technological process of documenting information and an object of legal regulation // State and Law. 2003. - No. 1. - S. 92-93. 
8. Marchenko A.A. Preliminary considerations // http: // www. libertarium.ru/l-eldoc-mar.

9. Semiletov S.I. Electronic document as a product of the technological process of documenting information and an object of legal regulation // State and Law. 2003. - No. 1. - P. 95.

10. Konyavsky V.A., Gadasin. V.A. Systemic difference between traditional and electronic documents // http: //www/accord.ru/index_otl.html.

11. A. V. Tkachev The use of electronic (computer) documents as evidence documents and written evidence in procedural relations // Criminalist Library: scientific journal. No. 5 (10). M., 2013.p. 130. 